\title{
A novel octanuclear Mn(III) aggregate as a single-molecule magnet $\uparrow$
}

\author{
Meenal D. Godbole, ${ }^{a}$ Olivier Roubeau, ${ }^{* b}$ Rodolphe Clérac, ${ }^{b}$ Huub Kooijman, ${ }^{c}$ Anthony L. Spek ${ }^{c}$ and \\ Elisabeth Bouwman*a
}

Received (in Cambridge, UK) 23rd March 2005, Accepted 25th May 2005

First published as an Advance Article on the web 15th June 2005

DOI: $10.1039 / b 504116 c$

The novel octanuclear cluster $\left[\mathrm{Mn}_{8} \mathrm{O}_{2}(\mathrm{OH})_{2}(\mathrm{OMe})_{12}(\mathrm{OAc})_{2}-\right.$ (Mesalim) $)_{4}$ (1) presents SMM behaviour with a relatively high experimental energy barrier $\left(\Delta_{\mathrm{eff}} / k_{\mathrm{B}}=36.0 \mathrm{~K}\right)$ as shown by its dc and ac magnetic properties.

The ability of a molecule to act as a nano-magnet is of potential interest for future data-storage applications. Such a molecule, commonly named a single-molecule magnet (SMM), ${ }^{1,2}$ displays slow relaxation of its magnetization and functions as a magnet below its blocking temperature $\left(T_{\mathrm{B}}\right)$. The relaxation time of the magnetization, $\tau$, is thermally activated and results mainly from the spin ground state $\left(S_{\mathrm{T}}\right)$ and the uni-axial anisotropy $(D)$ of the molecule. In this regime, the theoretical energy barrier $\Delta$ is equal to $|D| S_{\mathrm{T}}^{2}$ or $|D|\left(S_{\mathrm{T}}^{2}-1 / 4\right)$ for integer and half-integer $S_{\mathrm{T}}$, respectively. Although many efforts have been made to increase $\Delta$ and thus $T_{\mathrm{B}}$, the first family of SMMs, $\left[\mathrm{Mn}_{12} \mathrm{O}_{12}(\mathrm{OR})_{12}\left(\mathrm{H}_{2} \mathrm{O}\right)_{4}\right]$, still displays so far the highest blocking temperatures. ${ }^{1,2}$ Synthetic strategies in this field of research mostly rely on obtaining cores of transition metal ions with a high-spin ground state and a high single-ion anisotropy, using for example $\mathrm{Mn}$ (III) or Fe(III) linked through oxo- and/or carboxylato groups. ${ }^{3,4}$ Organic chelating ligands, which can act as an outer shell to these cores, are efficient in varying core size and key structural parameters such as JahnTeller (JT) elongation axis orientations (thus $D$ ), and the magnetic interaction scheme (thus $S_{\mathrm{T}}$ ). ${ }^{5}$ We have been using HMesalim (see Scheme 1) as a chelating ligand for studying the manganese and iron complexes in oxidation catalysis and report here on the structure and properties of $\left[\mathrm{Mn}_{8} \mathrm{O}_{2}(\mathrm{OH})_{2}(\mathrm{OMe})_{12}(\mathrm{OAc})_{2}\right.$ (Mesalim) $)_{4}$ (1), which is the first compound of this ligand to behave as an SMM.

The reaction of HMesalim with manganese(II) acetate in a $2: 1$ ratio in a methanol-ether mixture yields the mononuclear complex $\left[\mathrm{Mn}(\mathrm{Mesalim})_{2}(\mathrm{OAc})(\mathrm{MeOH})\right] \cdot \mathrm{MeOH}(2)$, the crystal structure of<smiles>COC(=N)c1ccccc1O</smiles>

Scheme 1 The ligand HMesalim (methyl salicylimidate).

${ }^{a}$ Leiden Institute of Chemistry, Gorlaeus Laboratories, Leiden University, P.O. Box 9502, 2300 RA Leiden, The Netherlands. E-mail: bouwman@chem.leidenuniv.nl; Fax: +31 715274551 ${ }^{b}$ Centre de Recherche Paul Pascal CNRS UPR-8641, 115 avenue du Dr. A. Schweitzer, 33600 Pessac, France.E-mail: roubeau@crppbordeaux.cnrs.fr; Fax: +33556845600

${ }^{c}$ Bijvoet Center for Biomolecular Research, Crystal and Structural Chemistry, Utrecht University, The Netherlands

$\dagger$ Electronic supplementary information (ESI) available: semilog plot of $\chi_{\mathrm{M}} T$ vs. $T$, Cole-Cole plots. See http://dx.doi.org/10.1039/b504116c which has recently been reported. ${ }^{6}$ The octanuclear complex 1 was obtained as brown crystals by very slow evaporation of a dilute methanol solution of $\mathbf{2}$ over a period of three months. $\$$ Despite the low yields and longer times necessary for the crystallisation, the complex can be readily reproduced. Structural analysis§ shows the compound to consist of neutral octanuclear clusters (see Fig. 1), containing eight $\mathrm{Mn}(\mathrm{III})$ metal ions, two $\mu_{4}-\mathrm{O}^{2-}$, two $\mu_{3}-\mathrm{OH}^{-}$, ten $\mu-\mathrm{OMe}^{-}$, two terminal $\mathrm{OMe}^{-}$, two $\mathrm{OAc}^{-}$anions and four terminal anionic Mesalim ligands. An alternative description of the complex would involve two terminal $\mathrm{MeOH}$ instead of $\mathrm{OMe}^{-}$and two $\mathrm{O}^{2-}$ instead of $\mathrm{OH}^{-}$. These descriptions differ only in the position of two $\mathrm{H}$ atoms. These $\mathrm{H}$ atoms are most likely disordered and could not be located on difference Fourier maps. The molecule has non-crystallographic inversion symmetry (RMS deviation $0.109 \AA$ ). All manganese ions are in a pseudo-octahedral environment. The four peripheral ones (Mn1, Mn4, Mn7 and $\mathrm{Mn} 8)$ are in an $\mathrm{NO}_{5}$ coordination sphere, whereas the four central manganese ions (Mn2, Mn3, Mn5 and Mn6) have $\mathrm{MnO}_{6}$ chromophores. Complex 1 can be described as consisting of six $\left[\mathrm{Mn}_{3} \mathrm{O}_{4}\right]$ partial cubane units that are commonly encountered in high nuclearity manganese compounds, the two central units sharing one face. Furthermore, each of the central partial cubane units shares a face with two of the four remaining units. The two terminal $\mathrm{MeO}^{-}$ions, the bridging acetates and the anionic

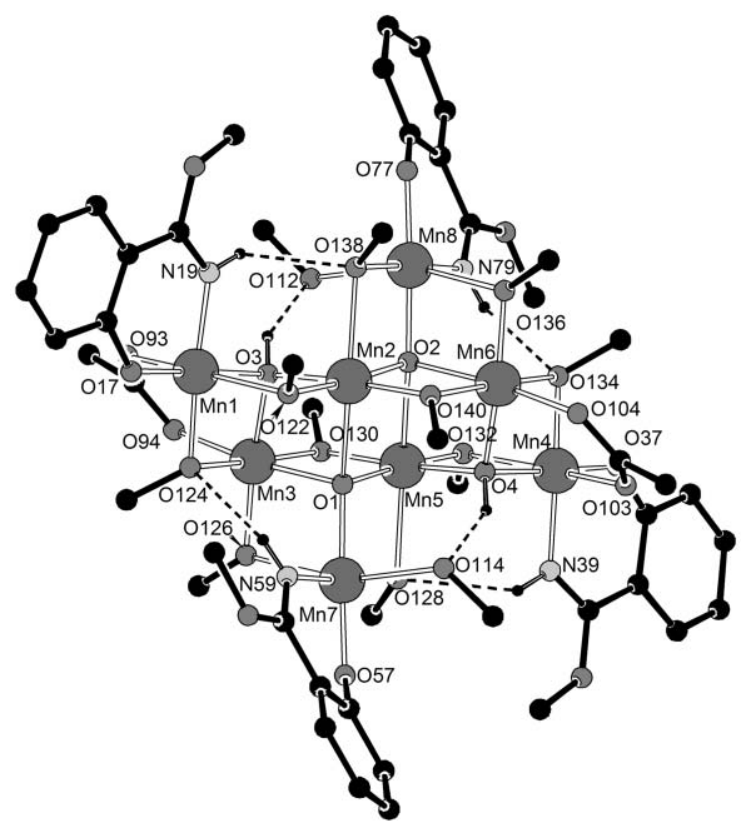

Fig. 1 A PLUTON view of the structure of complex 1. H atoms not participating in $\mathrm{H}$-bonds (dashed lines) are omitted for clarity. 
Mesalim ligands are not involved in the formation of partial cubanes, and complete the coordination sphere around the manganese ions. Pairs of neighbouring manganese ions are connected by two $\mathrm{O}$ bridges, the exception being the Mn1-Mn3 pair, and its pseudo-symmetrical counterpart Mn4-Mn6 pair, which have an additional acetate bridge resulting in shorter

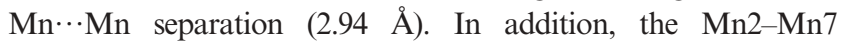
and $\mathrm{Mn} 5-\mathrm{Mn} 8$ pairs are connected by a single unique asymmetric $\mu_{4}-\mathrm{O}^{2-}$ bridge (for the $\mathrm{Mn} 2-\mathrm{Mn} 7$ pair: $\mathrm{Mn}-\mathrm{O}=2.295(5)$ and 1.911(5) $\AA, \mathrm{Mn}-\mathrm{O}-\mathrm{Mn}=150.7(3)^{\circ}$, for Mn5-M8: $\mathrm{Mn}-\mathrm{O}=2.305(5)$ and 1.921(5) $\left.\AA, \mathrm{Mn}-\mathrm{O}-\mathrm{Mn}=150.8(3)^{\circ}\right)$ resulting in a longer $\mathrm{Mn} \cdots \mathrm{Mn}$ separation (4.070(2) $\AA$ ). All Mn(III) ions present elongated JT axes, which is known to result in a negative value of the axial zero field splitting parameter. ${ }^{7}$ In the central Mn2-Mn5 pair both bridging $\mathrm{O}$ atoms lie along the JT elongation axis of either of the Mn ions, the JT axes of the two $\mathrm{Mn}$ (III) ions being parallel with each other in this case (Fig. 2) (short Mn-O bond, 1.957(6) and 1.967(6) A, long Mn-O bond, 2.295(5) and 2.305(5) $\AA, \mathrm{Mn} 2 \cdots \mathrm{Mn} 5=3.248(2) \AA)$. In contrast, in the rest of the pairs (Mn $\cdots$ Mn separations of 3.02 to $3.09 \AA$ ) only one of the two bridging $\mathrm{O}$ atoms lies on the JT axis of one of the manganese ions resulting in the orthogonality of the JT axes. The JT axes of Mn1, Mn4, Mn7 and Mn8 are all parallel to each other; the JT axis of Mn2 is parallel to Mn5 while the JT axis of Mn3 is parallel to Mn6. Each of the above three groups of parallel JT axes are approximately orthogonal to the other two orientations. A resulting significant magnetic anisotropy can thus be expected. Remarkably, the outer manganese ions do not have their JT axes along the bonds to ligand oxygen or nitrogen atoms. It is worth noting that octanuclearity in purely $\mathrm{Mn}(\mathrm{III})$ oxo/carboxylato compounds is still rather rare, ${ }^{8}$ and the core present in $\mathbf{1}$ has not been reported so far. However, the central $\mathrm{Mn}_{6}$ core resembles that of a hexamanganese complex reported by Tuchagues et al. ${ }^{9}$

Results of dc magnetic susceptibility measurements on a polycrystalline sample of $\mathbf{1}$ are depicted in Fig. 3. The $\chi_{\mathrm{M}} T$ product increases from $24.2 \mathrm{~cm}^{3} \mathrm{~K} \mathrm{~mol}^{-1}$ at $300 \mathrm{~K}$ to reach a maximum of $108.3 \mathrm{~cm}^{3} \mathrm{~K} \mathrm{~mol}^{-1}$ at $6.7 \mathrm{~K}$, and then decreases to $78.4 \mathrm{~cm}^{3} \mathrm{~K} \mathrm{~mol}^{-1}$ at $1.83 \mathrm{~K}$. This behaviour indicates the presence of dominant ferromagnetic interactions among the $\left[\mathrm{Mn}_{8}\right]$ core, yielding a high-spin ground state. The value of $\chi_{\mathrm{M}} T$ at $300 \mathrm{~K}$ is

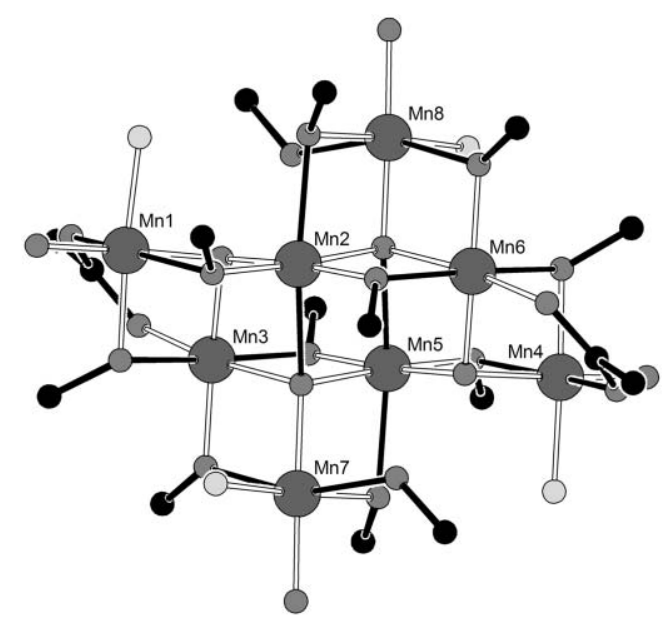

Fig. 2 A PLUTON view of the core of complex 1. JT axes of all Mn(III) ions are emphasized in black.

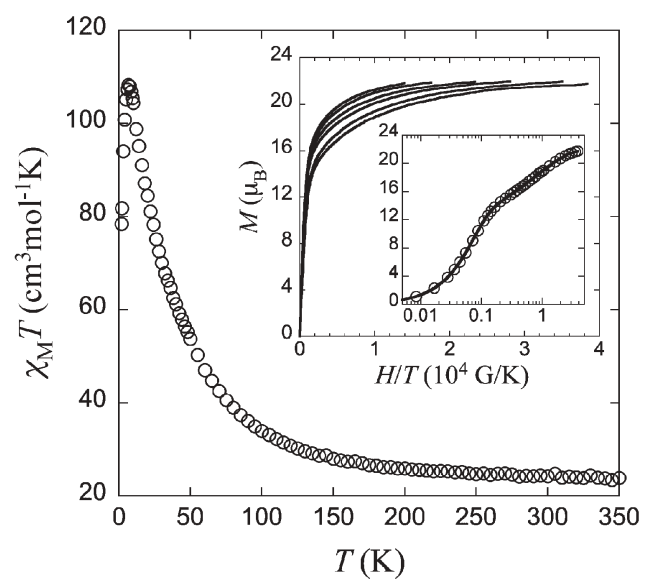

Fig. 3 Plot of $\chi_{\mathrm{M}} T$ vs. $T$ at $0.1 \mathrm{~T}$. Inset: Reduced magnetization data as normal and semilog plots.

in good agreement with the spin only value for eight uncoupled $S=2$ spins, e.g. $24 \mathrm{~cm}^{3} \mathrm{~K} \mathrm{~mol}^{-1}$. Owing to the size of the molecule and the symmetry of the interaction scheme, it was neither possible to apply the Kambe method $^{10}$ nor a simple numerical approach to evaluate the exchange parameters between the Mn(III) ions. Nevertheless, the central Mn2-Mn5 interaction is expected to be ferromagnetic, as observed in such out-of-plane Mn(III) dimeric units, ${ }^{11}$ while the other exchange parameters are likely to be much weaker, of either sign. The reduced magnetization plot presents a very steep increase of $M$ at low fields up to a value of $c a .16 \mu_{\mathrm{B}}$ (in agreement with a $S_{\mathrm{T}}=8$ ground state), and then a slower increase, without reaching saturation at the highest field $(7 \mathrm{~T})$. This feature and the lack of low temperature saturation of the $\chi_{\mathrm{M}} T$ product (see the semilog $\chi_{\mathrm{M}} T$ vs. $T$ plot in supplementary data, Fig. S1 $\dagger$ ) indicate that even at the experimental lowest temperature low-lying excited states are populated. Moreover, the fact that the data are not superimposed clearly indicates the presence of magnetic anisotropy (zero field splitting, ZFS). This behaviour could not be reproduced by considering that only the ground state is populated at the temperatures studied $(1.83-4 \mathrm{~K})$, as is usually done to ascertain the value of the spin ground state and its anisotropy parameter $D .{ }^{12}$ Hence the most probable situation is that the spin ground state of complex 1 would be $S_{\mathrm{T}}=8$, but with very low-lying excited spin-states. An $S_{\mathrm{T}}=8$ ground state would be in agreement with a central Mn2-Mn5 ferromagnetic interaction, the rest of the $\mathrm{Mn}$ pairs being antiferromagnetically coupled. The fall of $\chi_{\mathrm{M}} T$ at low temperatures is then either a consequence of intramolecular competing antiferromagnetic and ferromagnetic interactions among these pairs (note that no significant inter-molecular interactions have been observed in the crystal structure) and/or of magnetic anisotropy (ZFS).

Slow relaxation of the magnetization was studied using ac techniques. The frequency dependence of the ac susceptibility on a polycrystalline sample of $\mathbf{1}$ was measured at different temperatures down to $1.83 \mathrm{~K}$ under zero applied dc field (Fig. 4). As expected for a SMM, the ac susceptibility and especially its out-of-phase component are strongly frequency-dependent indicative of a slow magnetization-relaxation phenomenon. Remarkably, blocking temperatures can be observed in $\mathbf{1}$ at reasonable temperatures, i.e. above $3 \mathrm{~K}$ for frequencies higher than $500 \mathrm{~Hz}$. Fig. 4 as well as 


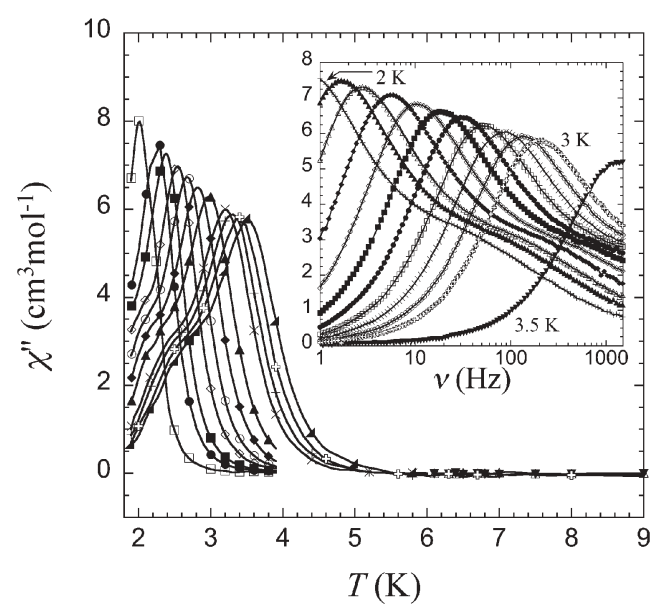

Fig. 4 Plot of $\chi^{\prime \prime} v s . T$ at $0 \mathrm{~T}$ dc field (frequencies from left to right: 1, 5, 10, 25, 50, 100, 199, 498, 699, 997 and $1488 \mathrm{~Hz}$ ). Inset: Plot of $\chi^{\prime \prime} v s . v$ at $0 \mathrm{~T}$ dc field at temperatures between 2 and $3 \mathrm{~K}$ and at $3.5 \mathrm{~K}$.

Cole-Cole plots (see supplementary material, Fig. S2†) indicate the presence of a second relaxation mode at higher frequencies, aside from the main one observed, which could be due to a small impurity or to intermolecular effects. ${ }^{13}$ From the data in Fig. 4, the main relaxation time $\tau$ can be determined from the maximum of $\chi^{\prime \prime}$ as a function of both temperature and frequency. In the temperature domain studied the relaxation time follows the Arrhénius law with $\Delta / k_{\mathrm{B}}=36.0 \mathrm{~K}$ and $\tau_{0}=4.39 \times 10^{-9} \mathrm{~s}$ (for $T>2.1 \mathrm{~K}$, see Fig. 5), indicating that the relaxation is thermally activated. As in many SMMs, it is likely that $\Delta$ corresponds in fact to an effective barrier, resulting from the short-cut of the thermal barrier by quantum tunnelling of magnetization. In $\mathbf{1}$, the experimental energy barrier $\Delta_{\mathrm{eff}} / k_{\mathrm{B}}=36.0 \mathrm{~K}$ remains relatively large. The octanuclear core of $\mathbf{1}$ is thus a new addition to the still quite small number of metal ion cores in which this behaviour has been observed at reasonably high temperatures.

In summary, a novel octanuclear Mn(III) SMM cluster has been isolated with the ligand HMesalim, which thus represents an interesting new entry in the small list of chelating ligands used so far in this field. The study of the magnetic properties of the novel cluster has revealed a rather high-energy barrier allowing the observation of thermally activated relaxation above $3 \mathrm{~K}$.

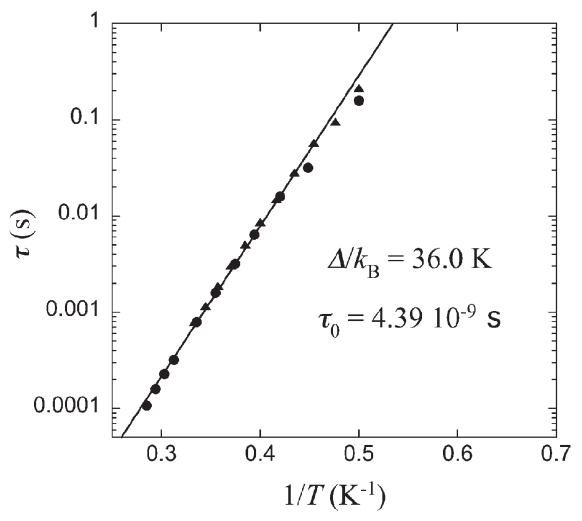

Fig. 5 Plot of $\tau$ vs. $T^{-1}$ determined by ac measurements (full circles: temperature data, full triangles: frequency data). The solid line represents the Arrhenius fit.
This work has been carried out within the framework of the Council for Chemical Sciences of the Netherlands Foundation for Scientific Research (CW-NWO), through a grant from the special program "Aspasia". The authors thank Prof. Jan Reedijk for stimulating discussions. Financial support from the University Bordeaux 1, the CNRS and the Région Aquitaine is gratefully acknowledged.

\section{Notes and references}

* Elemental analysis of crystals of 1: Anal. calc. for $\left[\mathrm{Mn}_{8} \mathrm{O}_{2}(\mathrm{OH})_{2}(\mathrm{OMe})_{12}(\mathrm{OAc})_{2}(\text { mesalim })_{4}\right]$ : C, 36.1; H, 4.8; N, 3.5. Found: C, 35.88; H, 4.61; N, 3.50. IR, / $\mathrm{cm}^{-1}: 2907$ (b, m), 2809 (m), 1616 (s), $1550(\mathrm{~ms}), 1544$ (ms), 1452 (ms), 1398 (s), $1336(\mathrm{~ms}), 1324$ (ms), 1266 (ms), $1221(\mathrm{~ms}), 1063(\mathrm{~s}), 1035(\mathrm{~s}), 867(\mathrm{~ms}), 769(\mathrm{~ms}), 550(\mathrm{vs})$.

$\S$ Crystal data for $1, \mathrm{C}_{48} \mathrm{H}_{76} \mathrm{Mn}_{8} \mathrm{~N}_{4} \mathrm{O}_{28}, M_{\mathrm{r}}=1596.65$, monoclinic, space group $P 2_{1} / c$ (No. 14), $a=9.753(3) \AA, b=27.559(6) \AA, c=25.860(8) \AA$, $\beta=115.621(13)^{\circ}, V=6267(3) \AA^{3}, Z=4, T=150 \mathrm{~K}$. Multiscan based absorption correction ( $T$ range $0.787-0.808)$. Refinement of 811 parameters against 11344 reflections gave $\mathrm{w} R\left(F^{2}\right)=0.1582, R(F>\sigma(F)) 0.0816$. CCDC 261986. See http://dx.doi.org/10.1039/b504116c for crystallographic data in CIF or other electronic format.

1 A. Caneschi, D. Gatteschi, R. Sessoli, A. L. Barra, L. C. Brunel and M. Guillot, J. Am. Chem. Soc., 1991, 113, 15, 5873; G. Christou, D. Gatteschi, D. N. Hendrickson and R. Sessoli, MRS Bull., 2000, 25, 66; D. Gatteschi and R. Sessoli, Angew. Chem., 2003, 115, 278, Angew. Chem., Int. Ed., 2003, 42, 268; S. K. Ritter, Chem. Eng. News, 2004, 82, $50,29$.

2 R. Sessoli, H.-L. Tsai, A. R. Schake, S. Wang, J. B. Vincent, K. Folting, D. Gatteschi, G. Christou and D. N. Hendrickson, J. Am. Chem. Soc., 1993, 115, 1804.

3 See for example: J. Yoo, E. K. Brechin, A. Yamaguchi, M. Nakano, J. C. Huffman, A. L. Maniero, L. C. Brunel, K. Awaga, H. Ishimoto, G. Christou and D. N. Hendrickson, Inorg. Chem., 2000, 39, 3615; J. P. Price, S. R. Batten, B. Moubaraki and K. S. Murray, Chem. Commun., 2002, 762; M. Soler, W. Wernsdorfer, K. Folting, M. Pink and G. Christou, J. Am. Chem. Soc., 2004, 126, 2156.

4 See for example: D. Gatteschi, R. Sessoli and A. Cornia, Chem. Commun., 2000, 725; J. C. Goodwin, R. Sessoli, D. Gatteschi, W. Wernsdorfer, A. K. Powell and S. L. Heath, J. Chem. Soc., Dalton Trans., 2000, 1835.

5 See for example: C. Boskovic, W. Wernsdorfer, K. Folting, J. C. Huffman, D. N. Hendrickson and G. Christou, Inorg. Chem., 2002, 41, 5107; N. C. Harden, M. A. Bolcar, W. Wernsdorfer, K. A. Abboud, W. E. Streib and G. Christou, Inorg. Chem., 2003, 42, 7067; L. M. Wittick, K. S. Murray, B. Moubaraki, S. R. Batten, L. Spiccia and K. J. Berry, Dalton Trans., 2004, 1003.

6 M. D. Godbole, M. Kloskowski, R. Hage, A. Rompel, A. M. Mills, A. L. Spek and E. Bouwman, Eur. J. Inorg. Chem., 2005, 305.

7 H. J. Gerritsen and E. S. Sabinsky, Phys. Rev., 1963, 132, 1507.

8 E. Libby, K. Folting, C. J. Huffman, J. C. Huffman and G. Christou, Inorg. Chem., 1993, 32, 2549; S. Wang, H.-L. Tsai, K. Folting, J. D. Martin, D. N. Hendrickson and G. Christou, Chem. Commun., 1994, 671; H.-L. Tsai, S. Wang, K. Folting, W. E. Streib, D. N. Hendrickson and G. Christou, J. Am. Chem. Soc., 1995, 117, 2503; L. F. Jones, E. K. Brechin, D. Collinson, J. Raftery and S. J. Teat, Inorg. Chem., 2003, 42, 691; G. Rajaraman, M. Murugesu, E. C. Sañudo, M. Soler, W. Wernsdorfer, M. Helliwell, C. Muryn, J. Raftery, S. J. Teat, G. Christou and E. K. Brechin, J. Am. Chem. Soc., 2004, 126, 15445.

9 M. Verelst, J. C. Daran and J.-P. Tuchagues, J. Chem. Soc., Chem. Commun., 1995, 2155.

10 K. Kambe, J. Phys. Soc. Jpn., 1950, 5, 48.

11 H. Miyasaka, R. Clérac, T. Ishii, H.-C. Chang, S. Kitagawa and M. Yamashita, J. Chem. Soc., Dalton Trans., 2002, 1528.

12 J. B. Vincent, C. Christmas, H. R. Chang, Q. Li, P. D. W. Boyd, J. C. Huffman, D. N. Hendrickson and G. Christou, J. Am. Chem. Soc., 1989, 111, 2086.

13 C. Boskovic, R. Bircher, P. L. W. Tregenna-Piggott, H. U. Güdel, C. Paulsen, W. Wernsdorfer, A.-L. Barra, E. Khatsko, A. Neels and H. Stoeckli-Evans, J. Am. Chem. Soc., 2003, 125, 14046. 